\title{
COMPLETE CONSTANT GAUSSIAN CURVATURE SURFACES IN THE MINKOWSKI SPACE AND HARMONIC DIFFEOMORPHISMS ONTO THE HYPERBOLIC PLANE
}

\author{
Jose A. Gálvez,* Antonio Martínez* And Francisco Milán**
}

(Received September 3, 2001, revised June 27, 2002)

\begin{abstract}
We complete the global classification of spacelike surfaces in the Minkowski three-space with constant Gaussian curvature in terms of harmonic diffeomorphisms onto the hyperbolic plane. A harmonic representation of them is also obtained.
\end{abstract}

1. Introduction. Similarly to the Euclidean case, the study of the Gauss map provides us with an important tool to investigate the properties of surfaces in the Minkowski 3-space $\boldsymbol{L}^{3}$. From this standpoint, Kobayashi [11], [12] and Akutagawa and Nishikawa [2] obtain Lorentzian versions of the classical Enneper-Weierstrass and Kenmotsu representations, that play a fundamental role in the research of maximal and constant mean curvature surfaces, respectively. In particular, they find numerous examples of surfaces with constant mean curvature in $\boldsymbol{L}^{3}$ and extend the well-known result that the Gauss map of these surfaces is harmonic, see [15] and [16].

Using this fact, from a global point of view, Au and Wan [20], [21] obtain the classification of complete spacelike surfaces with constant mean curvature in $\boldsymbol{L}^{3}$, using one-to-one harmonic maps into the hyperbolic plane $\boldsymbol{H}_{+}^{2}$. Moreover, an important consequence of that result, answering a question of Eells and Lemaire (see [6]), is the existence of harmonic maps of rank 2 into $\boldsymbol{H}_{+}^{2}$, see also [1] and [4]. In particular, they find harmonic diffeomorphisms from the unit disk $\boldsymbol{D}$ onto $\boldsymbol{H}_{+}^{2}$ and pose the question whether there is some harmonic diffeomorphism from the Euclidean plane onto the hyperbolic plane, see [3].

The main goal of this paper is to give a similar representation for surfaces in $\boldsymbol{L}^{3}$, using the Gauss map and the conformal structure given by the second fundamental form. In our method, it is deduced that the Gaussian curvature is a negative constant if and only if the Gauss map is harmonic with respect to the Riemannian metric given by the second fundamental form. Thus, we obtain as a main result the classification of complete spacelike surfaces with negative constant Gaussian curvature in $\boldsymbol{L}^{3}$, in terms of harmonic diffeomorphisms onto the hyperbolic plane. Moreover, since there do not exist isometric immersions from the sphere into $\boldsymbol{L}^{3}$, our result completes the global classification of spacelike surfaces with constant Gaussian curvature in the Minkowski 3-space (see [5] and [9]).

2000 Mathematics Subject Classification. Primary 53C42; Secondary 53C43, 58E20.

Key words and phrases. Gaussian curvature, Weierstrass representation, harmonic maps.

*Partly supported by DGICYT Grant No. BFM2001-3318. 
Finally, we obtain an interesting relation between harmonic diffeomorphisms onto $\boldsymbol{H}_{+}^{2}$ and a Monge-Ampère equation in a bounded convex domain.

2. Spacelike surfaces in $\boldsymbol{L}^{3}$. Let $\boldsymbol{L}^{3}$ be the Minkowski 3-space endowed with the canonical linear coordinates $\left(x_{1}, x_{2}, x_{3}\right)$ and the scalar product $\langle$,$\rangle given by the quadratic$ form $d x_{1}^{2}+d x_{2}^{2}-d x_{3}^{2}$. Associated with this metric one has the exterior product of two vectors $u, v \in \boldsymbol{L}^{3}$ given as the unique vector $u \times v$ such that $\langle u \times v, w\rangle=-\operatorname{det}(u, v, w)$ for all $w \in \boldsymbol{L}^{3}$, where det denotes the usual determinant.

Consider the unit pseudosphere

$$
\boldsymbol{H}^{2}=\left\{\left(x_{1}, x_{2}, x_{3}\right) \in \boldsymbol{L}^{3} \mid x_{1}^{2}+x_{2}^{2}-x_{3}^{2}=-1\right\},
$$

which is a two-sheeted hyperboloid, with constant Gaussian curvature -1 with respect to the induced metric. We introduce complex coordinates on $\boldsymbol{H}^{2}$ by using the stereographic mappings $\psi_{1}: \boldsymbol{H}^{2}-\{(0,0,-1)\} \rightarrow \boldsymbol{C}$ and $\psi_{2}: \boldsymbol{H}^{2}-\{(0,0,1)\} \rightarrow \boldsymbol{C}$ defined, respectively, as

$$
\psi_{1}\left(x_{1}, x_{2}, x_{3}\right)=\frac{x_{1}-i x_{2}}{1+x_{3}}, \quad \psi_{2}\left(x_{1}, x_{2}, x_{3}\right)=\frac{x_{1}+i x_{2}}{1-x_{3}} .
$$

If we take $\boldsymbol{H}_{+}^{2}=\boldsymbol{H}^{2} \cap\left\{x_{3}>0\right\}$, then $\psi_{1}: \boldsymbol{H}_{+}^{2} \rightarrow \boldsymbol{D}=\{w \in \boldsymbol{C}|| w \mid<1\}$ is a biholomorphic map and the induced metric on $\boldsymbol{H}_{+}^{2}$ can be written as

$$
d s_{P}^{2}=\frac{4|d w|^{2}}{\left(1-|w|^{2}\right)^{2}},
$$

which is called the Poincaré metric.

Let us consider a connected smooth surface $S$ and a spacelike immersion $x: S \rightarrow L^{3}$ with negative Gaussian curvature with respect to the induced Riemannian metric $I$. Since $S$ is orientable, we choose the orientation on $S$ given by a unit normal vector field $N: S \rightarrow \boldsymbol{H}^{2}$ such that the second fundamental form $I I=\langle d x, d N\rangle$ is positive definite. Up to the isometry $\varphi$ of $\boldsymbol{L}^{3}, \varphi\left(x_{1}, x_{2}, x_{3}\right)=\left(x_{1}, x_{2},-x_{3}\right)$, we will supose throughout this section that $N(S) \subseteq$ $\boldsymbol{H}_{+}^{2}$.

Thus, from now on $S$ will be considered as a Riemann surface with the conformal structure induced by $I I$. If $z=u+i v$ is a conformal parameter, then

$$
\begin{aligned}
& I=E d u^{2}+2 F d u d v+G d v^{2}, \\
& I I=e\left(d u^{2}+d v^{2}\right),
\end{aligned}
$$

with $e>0$ and the Weingarten equations (see p. 157 in [22]) state that

(2) $\quad N_{u}=\frac{\partial N}{\partial u}=\frac{e}{E G-F^{2}}\left(G x_{u}-F x_{v}\right), \quad N_{v}=\frac{\partial N}{\partial v}=\frac{e}{E G-F^{2}}\left(-F x_{u}+E x_{v}\right)$.

Let us denote by $g=\psi_{1} \circ N: S \rightarrow \boldsymbol{D}$ the composition of the usual stereographic projection with the Gauss map $N$, that is,

$$
g=\frac{N_{1}-i N_{2}}{1+N_{3}},
$$

where $N=\left(N_{1}, N_{2}, N_{3}\right)$. We will also call it the Gauss map of the immersion. 
THEOREM 1. Let $x: S \rightarrow \boldsymbol{L}^{3}$ be a spacelike immersion with negative Gaussian curvature $K<0, g: S \rightarrow D$ its Gauss map and $z=u+i v$ a conformal parameter. Then

$$
\begin{aligned}
\frac{\partial x_{1}}{\partial z} & =\frac{-\left(1+\bar{g}^{2}\right) g_{z}+\left(1+g^{2}\right) \bar{g}_{z}}{\sqrt{-K}(1-g \bar{g})^{2}} \\
\frac{\partial x_{2}}{\partial z} & =-i \frac{\left(1-\bar{g}^{2}\right) g_{z}+\left(1-g^{2}\right) \bar{g}_{z}}{\sqrt{-K}(1-g \bar{g})^{2}} \\
\frac{\partial x_{3}}{\partial z} & =2 \frac{-\bar{g} g_{z}+g \bar{g}_{z}}{\sqrt{-K}(1-g \bar{g})^{2}}
\end{aligned}
$$

where $x=\left(x_{1}, x_{2}, x_{3}\right), \partial / \partial z=(1 / 2)(\partial / \partial u-i \partial / \partial v)$ and the complex conjugation is denoted by bar.

Proof. From (1) and (2), the Gauss map $N$ and the Gaussian curvature $K$ are given by

$$
N=\frac{x_{u} \times x_{v}}{\sqrt{E G-F^{2}}}, \quad K=\frac{-e^{2}}{E G-F^{2}} .
$$

Hence, one obtains

$$
N \times N_{u}=-\sqrt{-K} x_{v}, \quad N \times N_{v}=\sqrt{-K} x_{u}
$$

and

$$
x_{z}=\frac{i}{\sqrt{-K}} N \times N_{z} .
$$

From (3), noting that $\langle N, N\rangle=-1$, one then has

$$
N_{1}=\frac{g+\bar{g}}{1-g \bar{g}}, \quad N_{2}=i \frac{g-\bar{g}}{1-g \bar{g}}, \quad N_{3}=\frac{1+g \bar{g}}{1-g \bar{g}},
$$

from which (4) follows.

A straightforward computation then implies

COROLLARY 1. With the above notation, the first, second and third fundamental forms of the immersion are given, respectively, by

$$
\begin{aligned}
& I=\frac{4}{-K(1-g \bar{g})^{2}}\left(-g_{z} \bar{g}_{z} d z^{2}+\left(\left|g_{\bar{z}}\right|^{2}+\left|g_{z}\right|^{2}\right)|d z|^{2}-g_{\bar{z}} \bar{g}_{\bar{z}} d \bar{z}^{2}\right), \\
& I I=4 \frac{\left|g_{\bar{z}}\right|^{2}-\left|g_{z}\right|^{2}}{\sqrt{-K}(1-g \bar{g})^{2}}|d z|^{2}, \\
& I I I=\frac{4}{(1-g \bar{g})^{2}}\left(g_{z} \bar{g}_{z} d z^{2}+\left(\left|g_{\bar{z}}\right|^{2}+\left|g_{z}\right|^{2}\right)|d z|^{2}+g_{\bar{z}} \bar{g}_{\bar{z}} d \bar{z}^{2}\right) .
\end{aligned}
$$

Moreover, the mean curvature $H$ of the immersion satisfies

$$
H=\sqrt{-K} \frac{\left|g_{\bar{z}}\right|^{2}+\left|g_{z}\right|^{2}}{\left|g_{\bar{z}}\right|^{2}-\left|g_{z}\right|^{2}} .
$$

Concerning the structure equations of the immersion, we have 
THEOREM 2. If $x: S \rightarrow \boldsymbol{L}^{3}$ is a spacelike immersion with negative Gaussian curvature $K<0$, then the Gauss map $g$ satisfies the equation

$$
4 K\left(g_{z \bar{z}}+2 \bar{g} \frac{g_{z} g_{\bar{z}}}{1-g \bar{g}}\right)=K_{z} g_{\bar{z}}+K_{\bar{z}} g_{z} .
$$

Moreover, the Gaussian curvature is determined, up to multiplication by positive constants, by the Gauss map.

Proof. From Theorem 1 it follows that $\left(x_{j z}\right)_{\bar{z}}=\left(x_{j}\right)_{z}, j=1,2,3$, if and only if the following hold

$$
\begin{aligned}
& \frac{8 K}{1-g \bar{g}}\left(\left(\bar{g}+\bar{g}^{3}\right) g_{z} g_{\bar{z}}-\left(g+g^{3}\right) \bar{g}_{z} \bar{g}_{\bar{z}}\right)+K_{z}\left(\left(1+g^{2}\right) \bar{g}_{\bar{z}}-\left(1+\bar{g}^{2}\right) g_{\bar{z}}\right) \\
& \quad+K_{\bar{z}}\left(\left(1+g^{2}\right) \bar{g}_{z}-\left(1+\bar{g}^{2}\right) g_{z}\right)+4 K\left(\left(1+\bar{g}^{2}\right) g_{z \bar{z}}-\left(1+g^{2}\right) \bar{g}_{z \bar{z}}\right)=0, \\
& \frac{-8 K}{1-g \bar{g}}\left(\left(\bar{g}-\bar{g}^{3}\right) g_{z} g_{\bar{z}}+\left(g-g^{3}\right) \bar{g}_{z} \bar{g}_{\bar{z}}\right)+K_{z}\left(\left(1-g^{2}\right) \bar{g}_{\bar{z}}+\left(1-\bar{g}^{2}\right) g_{\bar{z}}\right) \\
& \quad+K_{\bar{z}}\left(\left(1-g^{2}\right) \bar{g}_{z}+\left(1-\bar{g}^{2}\right) g_{z}\right)-4 K\left(\left(1-\bar{g}^{2}\right) g_{z \bar{z}}+\left(1-g^{2}\right) \bar{g}_{z \bar{z}}\right)=0, \\
& \quad \frac{8 K}{1-g \bar{g}}\left(\bar{g}^{2} g_{z} g_{\bar{z}}-g^{2} \bar{g}_{z} \bar{g}_{\bar{z}}\right)+K_{z}\left(g \bar{g}_{\bar{z}}-\bar{g} g_{\bar{z}}\right)+K_{\bar{z}}\left(g \bar{g}_{z}-\bar{g} g_{z}\right) \\
& \quad+4 K\left(\bar{g} g_{z \bar{z}}-g \bar{g}_{z \bar{z}}\right)=0 .
\end{aligned}
$$

Then, subtracting (11) and $2 g$ times (12) from (10), we obtain (E). Moreover, (E) together with its conjugated equation yields

$$
\begin{aligned}
& (\log (-K))_{z} g_{\bar{z}}+(\log (-K))_{\bar{z}} g_{z}=4\left(g_{z \bar{z}}+2 \bar{g} \frac{g_{z} g_{\bar{z}}}{1-g \bar{g}}\right), \\
& (\log (-K))_{z} \bar{g}_{\bar{z}}+(\log (-K))_{\bar{z}} \bar{g}_{z}=4\left(\bar{g}_{z \bar{z}}+2 g \frac{\bar{g}_{z} \bar{g}_{\bar{z}}}{1-g \bar{g}}\right) .
\end{aligned}
$$

Since $\left|g_{\bar{z}}\right|^{2}-\left|g_{z}\right|^{2}>0$, we then obtain

$$
(\log (-K))_{z}=\frac{4}{\left|g_{\bar{z}}\right|^{2}-\left|g_{z}\right|^{2}}\left(\bar{g}_{z} g_{z \bar{z}}-g_{z} \bar{g}_{z \bar{z}}-2 g_{z} \bar{g}_{z} \frac{g \bar{g}_{\bar{z}}-\bar{g} g_{\bar{z}}}{1-g \bar{g}}\right),
$$

from which it is clear that $K$ is determined, up to multiplication by positive constants, by $g$.

REMARK 1. The equation (L) is equivalent to (E). Also, (E) is satisfied if and only if (10), (11) and (12) are satisfied.

THEOREM 3. Let $S$ be a simply connected Riemann surface and $N: S \rightarrow \boldsymbol{H}_{+}^{2}$ a differentiable map. Then, a spacelike immersion $x: S \rightarrow \boldsymbol{L}^{3}$ with the Gauss map $N$ exists in such a way that the conformal structure on $S$ is induced by the second fundamental form if and only if the following hold.

$$
\left|g_{\bar{z}}\right|^{2}-\left|g_{z}\right|^{2}>0
$$




$$
\operatorname{Im}\left\{\frac{\partial}{\partial \bar{z}}\left(\frac{4}{\left|g_{\bar{z}}\right|^{2}-\left|g_{z}\right|^{2}}\left(\bar{g}_{z} g_{z \bar{z}}-g_{z} \bar{g}_{z \bar{z}}-2 g_{z} \bar{g}_{z} \frac{g \bar{g}_{\bar{z}}-\bar{g} g_{\bar{z}}}{1-g \bar{g}}\right)\right)\right\}=0,
$$

where $g$ is as in (3). Moreover, the immersion is unique, up to similarity transformations of $\boldsymbol{L}^{3}$, and can be recovered by the equations

$$
\begin{aligned}
& x_{1}=\operatorname{Re}\left(\int 2 \frac{-\left(1+\bar{g}^{2}\right) g_{z}+\left(1+g^{2}\right) \bar{g}_{z}}{\sqrt{-K}(1-g \bar{g})^{2}} d z\right)+c_{1}, \\
& x_{2}=\operatorname{Re}\left(\int-2 i \frac{\left(1-\bar{g}^{2}\right) g_{z}+\left(1-g^{2}\right) \bar{g}_{z}}{\sqrt{-K}(1-g \bar{g})^{2}} d z\right)+c_{2}, \\
& x_{3}=\operatorname{Re}\left(\int 4 \frac{-\bar{g} g_{z}+g \bar{g}_{z}}{\sqrt{-K}(1-g \bar{g})^{2}} d z\right)+c_{3},
\end{aligned}
$$

where

$$
\log (-K)=\operatorname{Re}\left(\int \frac{8}{\left|g_{\bar{z}}\right|^{2}-\left|g_{z}\right|^{2}}\left(\bar{g}_{z} g_{z \bar{z}}-g_{z} \bar{g}_{z \bar{z}}-2 g_{z} \bar{g}_{z} \frac{g \bar{g}_{\bar{z}}-\bar{g} g_{\bar{z}}}{1-g \bar{g}}\right) d z\right)+\lambda,
$$

$c_{1}, c_{2}, c_{3}, \lambda$ are real constants and the integral is taken along a path from a fixed point to the variable point.

PROOF. If $S$ is a Riemann surface with the conformal structure given by the second fundamental form of a spacelike immersion $x: S \rightarrow \boldsymbol{L}^{3}$, then $K<0$, so that both $\log (-K)$ and $\partial^{2}(\log (-K)) / \partial z \partial \bar{z}$ must be real. The result follows from Theorems 1 and 2 together with Corollary 1.

Conversely, since $S$ is simply connected, there exists $\varphi: S \rightarrow \boldsymbol{R}$ such that $K=-e^{\varphi}$ which satisfies (L) if and only if (14) is satisfied. Now, from Remark 1, it is easy to check that (L) (or equivalently, (E)) is the complete integrability condition for (4).

Moreover, if $x, y: S \rightarrow \boldsymbol{L}^{3}$ are two immersions as above with Gaussian curvature $K_{1}, K_{2}$, respectively, then $\left(\log \left(-K_{1}\right)\right)_{z}=\left(\log \left(-K_{2}\right)\right)_{z}$ and $K_{1}=r K_{2}$ for some positive constant $r$. Thus $y_{z}=\sqrt{r} x_{z}$ and $y=\sqrt{r} x+c, c \in \boldsymbol{L}^{3}$.

From the above theorem we obtain the following uniqueness result for immersions with negative Gaussian curvature,

Proposition 1. Let $S$ be a simply connected Riemann surface, and $x_{1}, x_{2}: S \rightarrow \boldsymbol{L}^{3}$ be two spacelike immersions with negative Gaussian curvature $K_{1}, K_{2}$ and the Gauss map $N_{1}, N_{2}: S \rightarrow \boldsymbol{H}_{+}^{2}$, respectively. Then the following conditions are equivalent:

(i) There exist a conformal equivalence $\varphi$ on $S$ and an orthocronal isometry $f$ of $\boldsymbol{L}^{3}$ (that is, an isometry preserving the temporal orientation of $\boldsymbol{L}^{3}$ ) such that $f \circ x_{1}=x_{2} \circ \varphi$.

(ii) There exist a conformal equivalence $\varphi$ on $S$ and an isometry $i$ of $\boldsymbol{H}_{+}^{2}$ such that $i \circ N_{1}=N_{2} \circ \varphi$ and $K_{1}=K_{2} \circ \varphi$.

ProOf. Since each isometry preserves the Gaussian curvature of an immersion, if $f \circ$ $x_{1}=x_{2} \circ \varphi$, then $K_{1}=K_{2} \circ \varphi$. Moreover, $d f\left(T_{x_{1}(p)} S\right)=T_{x_{2} \circ \varphi(p)} S$, where, for instance, $T_{x_{1}(p)} S$ denotes the tangent plane to the immersion $x_{1}$ at the point $x_{1}(p)$. Thus, if we consider $i$ as the restriction of $d f$ to $\boldsymbol{H}_{+}^{2}$, it is clear that $i \circ N_{1}=N_{2} \circ \varphi$. 
Conversely, given an isometry $i$ of $\boldsymbol{H}_{+}^{2}$, we consider $f^{\prime}$ as the extension of $i$ to an isometry of $\boldsymbol{L}^{3}$. If we take $x_{1}^{\prime}=f^{\prime} \circ x_{1}$, then from (ii), $N_{1}^{\prime}=N_{2} \circ \varphi$ and $K_{1}^{\prime}=K_{2} \circ \varphi$, where $N_{1}^{\prime}$ and $K_{1}^{\prime}$ are the Gauss map and Gaussian curvature of $x_{1}^{\prime}$. So, using Theorem 3, $d\left(x_{1}^{\prime}-x_{2} \circ \varphi\right)=0$. Therefore, $x_{1}^{\prime}=x_{2} \circ \varphi+c$ for some $c \in \boldsymbol{L}^{3}$ and the result follows.

REMARK 2. The above computations can be carried out in a similar way for an immersion with positive Gaussian curvature, considering $S$ as a Lorentz surface with the Lorentz metric induced by the second fundamental form.

3. Constant Gaussian curvature and harmonic maps. Let $x: S \rightarrow \boldsymbol{L}^{3}$ be a spacelike immersion with negative constant Gaussian curvature. Then, given a conformal parameter $z$ for its second fundamental form, we obtain, from Theorem 2 ,

$$
g_{z \bar{z}}+2 \bar{g} \frac{g_{z} g_{\bar{z}}}{1-g \bar{g}}=0
$$

that is, its Gauss map $N$ is harmonic into the hyperbolic plane.

Conversely, if $S$ is a simply connected Riemann surface and $N: S \rightarrow \boldsymbol{H}_{+}^{2}$ a harmonic local diffeomorphism then, from Theorem 3 , there exists, up to translations, a unique immersion with negative constant Gaussian curvature $K$ such that $N$ (or $-N$ ) is its Gauss map and its conformal structure is induced by the second fundamental form of the immersion.

Now, we define $\mathcal{A}_{K}$ as the set of all complete spacelike immersions in $\boldsymbol{L}^{3}$ with constant negative Gaussian curvature $K<0$, where congruent immersions are identified by isometries of $\boldsymbol{L}^{3}$, and $\mathcal{B}$ be the set of all harmonic diffeomorphisms from the unit disk $\boldsymbol{D}$ or the complex plane $\boldsymbol{C}$ onto the hyperbolic plane $\boldsymbol{H}_{+}^{2}$, where two harmonic diffeomorphisms $h_{1}, h_{2}$ are identified if there exist a conformal equivalence $\varphi$ on $\boldsymbol{D}$ or $\boldsymbol{C}$ and an isometry $i$ on $\boldsymbol{H}_{+}^{2}$ such that $h_{1}=i \circ h_{2} \circ \varphi$.

Then we obtain the following classification theorem.

THEOREM 4. There exists a bijective correspondence between $\mathcal{A}_{K}$ and $\mathcal{B}$ for all $K<0$. That is, complete spacelike surfaces with constant negative Gaussian curvature are classified in terms of harmonic diffeomorphisms from Riemann surfaces onto the hyperbolic plane.

PROOF. Note that without loss of generality we may suppose $K=-1$. Let $x: S \rightarrow \boldsymbol{L}^{3}$ be a complete spacelike immersion with constant Gaussian curvature -1 . Since its Gauss map $N$ is harmonic, we have

$$
\frac{\partial}{\partial \bar{z}}\left(\frac{g_{z} \bar{g}_{z}}{(1-g \bar{g})^{2}}\right)=0
$$

where $z$ is a conformal parameter for the second fundamental form $I I$ and $g=\psi_{1} \circ N$ or $\psi_{1} \circ(-N)$. Equivalently, from (8), the $(2,0)$-part of $I$ and $I I I$ are holomorphic with respect to $I I$ and the identity map Id : $(S, I I) \rightarrow(S, I)$ is a harmonic diffeomorphism.

On the other hand, noting that the immersion is complete, $x(S)$ is a graph on the entire plane, therefore $S$ is simply connected and there exist a conformal equivalence $\varphi$ from $S$ to 
$\boldsymbol{D}$ or $\boldsymbol{C}$ and an isometry $i:(S, I) \rightarrow \boldsymbol{H}_{+}^{2}$. In consequence, for any immersion $x$ as above we consider the harmonic diffeomorphism $i \circ \operatorname{Id} \circ \varphi^{-1}$ that is uniquely determined up to conformal equivalences on $\boldsymbol{D}$ or $\boldsymbol{C}$ and isometries on $\boldsymbol{H}_{+}^{2}$.

Thus, for any $x$ we consider an element of $\mathcal{B}, \Phi(x)=\left[i \circ \operatorname{Id} \circ \varphi^{-1}\right]$, given by the equivalence class of $i \circ \operatorname{Id} \circ \varphi^{-1}$. Moreover, if $y$ is another immersion such that $y=f \circ x$, for some isometry $f$ of $\boldsymbol{L}^{3}$, it is clear from Proposition 1 that $\Phi(x)=\Phi(y)$ and, hence, there exists a map $\bar{\Phi}: \mathcal{A}_{-1} \rightarrow \mathcal{B}$ given by $\bar{\Phi}([x])=\Phi(x)$.

Now, we consider two immersions $x_{j}: S_{j} \rightarrow \boldsymbol{L}^{3}, j=1,2$, such that $\left[x_{1}\right],\left[x_{2}\right] \in \mathcal{A}_{-1}$ and $\Phi\left(x_{1}\right)=\Phi\left(x_{2}\right)$. Then there exists a holomorphic diffeomorphism $\varphi: S_{1} \rightarrow S_{2}$ and an isometry $i:\left(S_{1}, I\right) \rightarrow\left(S_{2}, I\right)$ such that $\operatorname{Id}_{S_{1}}=i^{-1} \circ \operatorname{Id}_{S_{2}} \circ \varphi$. Therefore, $i=\varphi$ and $x_{1}, x_{2} \circ i: S_{1} \rightarrow \boldsymbol{L}^{3}$ are two immersions with the same induced metric and conformal structure for the second fundamental form. Since the Gaussian curvature of both immersions agree, from the theorem egregium (see pp. 156, 157 in [22]) the second fundamental form must be the same. Hence, $x_{1}$ and $x_{2} \circ i$ agree up to an isometry of $\boldsymbol{L}^{3},\left[x_{1}\right]=\left[x_{2}\right]$ and $\bar{\Phi}$ is one-to-one.

Let $\Delta$ be the unit disk or the complex plane and $h: \Delta \rightarrow \boldsymbol{H}_{+}^{2}$ a surjective harmonic diffeomorphism. From Theorem 3 there exists a spacelike immersion $y: \Delta \rightarrow \boldsymbol{L}^{3}$ with constant Gaussian curvature -1 such that $h$ is its Gauss map. On the other hand, the identity map $\operatorname{Id}_{\Delta}:\left(\Delta, I I_{y}\right) \rightarrow\left(\Delta, I_{y}\right)$ is harmonic, where, for instance, $I_{y}$ denotes the first fundamental form for $y$, and since $\Delta$ is simply connected, there exists an isometric immersion $i:\left(\Delta, I_{y}\right) \rightarrow \boldsymbol{H}_{+}^{2}$. Thus, from Theorem 3 there exists a spacelike immersion $x: \Delta \rightarrow \boldsymbol{L}^{3}$ with constant Gaussian curvature -1 such that $i \circ \mathrm{Id}_{\Delta}$ is its Gauss map. Moreover, from (8), $I I I_{x}=i^{*}\left(d s_{P}^{2}\right)=I_{y}$ gives $I I_{x}=I I_{y}$ and $I_{x}=I I I_{y}$. Therefore, $x$ is complete because of $I_{x}=\langle d h, d h\rangle=h^{*}\left(d s_{P}^{2}\right), \Phi(x)=[h]$ and $\bar{\Phi}$ is onto.

REMARK 3. From [1] together with the above theorem, it is clear that there exist infinitely many non-congruent isometric immersions from the hyperbolic plane in the Minkowski 3 -space. Moreover, each of them has the second fundamental form conformally equivalent to the unit disk.

On the other hand, the conjecture about the non-existence of harmonic diffeomorphisms from the complex plane onto the hyperbolic plane is equivalent to the non-existence of a complete spacelike immersion with negative constant Gaussian curvature and second fundamental form conformally equivalent to $\boldsymbol{C}$.

Concerning this, it is not difficult to prove the following result.

Proposition 2. Let $S$ be a simply connected surface and $x: S \rightarrow L^{3}$ be a spacelike immersion with constant negative Gaussian curvature $K<0$. If the mean curvature $H$ is bounded, then $S$, with the Riemann structure given by the second fundamental form, is conformal to the unit disk.

PROoF. From (8) the second fundamental form is conformal to the metric

$$
\sigma=\frac{4\left|g_{\bar{z}}\right|^{2}}{\sqrt{-K}(1-g \bar{g})^{2}}|d z|^{2} .
$$


Since $g$ is harmonic, the Gaussian curvature of $\sigma$ (see [17]) is given by

$$
K^{\sigma}=\sqrt{-K}\left(\frac{\left|g_{z}\right|^{2}}{\left|g_{\bar{z}}\right|^{2}}-1\right)
$$

Since $H$ is bounded, we obtain from (9) that there exists a constant $c<1$ such that $\left|g_{z}\right|^{2} /\left|g_{\bar{z}}\right|^{2} \leq c$ and $K^{\sigma} \leq \sqrt{-K}(c-1)<0$. Hence, using the Ahlfors-Schwarz lemma (see [10] p. 66), $S$ is conformally equivalent to the unit disk.

REMARK 4. The converse of the above proposition is not true. For instance, the surface $M_{0}$ in [9] is a complete spacelike immersion with constant negative Gaussian curvature -1 and non-bounded $H$, which is conformally equivalent to $\boldsymbol{D}$ with the structure given by its second fundamental form.

Now, we find a relation between harmonic diffeomorphisms onto $\boldsymbol{H}_{+}^{2}$ and a MongeAmpère equation in a bounded convex domain.

THEOREM 5. There exists a bijective correspondence between harmonic diffeomorphisms from the unit disk or the complex plane onto the hyperbolic plane (up to conformal equivalences in the domain) and the solutions of the Monge-Ampère equation

$$
\phi_{u u} \phi_{v v}-\phi_{u v}^{2}=\frac{1}{\left(1-u^{2}-v^{2}\right)^{2}}, \quad 0 \leq u^{2}+v^{2}<1 .
$$

Proof. Let $\Delta$ be $\boldsymbol{D}$ or $\boldsymbol{C}$ and $N: \Delta \rightarrow \boldsymbol{H}_{+}^{2}$ a surjective harmonic diffeomorphism. From Theorem 3, there exists a unique, up to translations, spacelike immersion $x: \Delta \rightarrow \boldsymbol{L}^{3}$ with constant negative Gaussian curvature -1 such that $N$ is its Gauss map and the Riemann structure on $\Delta$ is given by the second fundamental form. Then, we consider the Legendre transformation

$$
\phi(u, v)=u x_{1}+v x_{2}-x_{3},
$$

where $x=\left(x_{1}, x_{2}, x_{3}\right)$ is locally a graph on the $\left(x_{1}, x_{2}\right)$-plane, and the map given by $N=$ $\left(N_{1}, N_{2}, N_{3}\right) \rightarrow(u, v)=\left(N_{1} / N_{3}, N_{2} / N_{3}\right)$ is a diffeomorphism from $\boldsymbol{H}_{+}^{2}$ onto the unit disk.

Thus, $(u, v)$ are new parameters and a straightforward computation yields $\phi_{u}=x_{1}$, $\phi_{v}=x_{2}$ and

$$
D \phi_{u u}=\frac{\partial^{2} x_{3}}{\partial x_{2}^{2}}, \quad D \phi_{u v}=\frac{\partial^{2} x_{3}}{\partial x_{1} \partial x_{2}}, \quad D \phi_{v v}=\frac{\partial^{2} x_{3}}{\partial x_{1}^{2}},
$$

with $D=\left(\partial^{2} x_{3} / \partial x_{1}^{2}\right)\left(\partial^{2} x_{3} / \partial x_{2}^{2}\right)-\left(\partial^{2} x_{3} / \partial x_{1} \partial x_{2}\right)^{2}$.

Therefore, from the expresion of the Gaussian curvature

$$
K=-1=-\frac{\left(\partial^{2} x_{3} / \partial x_{1}^{2}\right)\left(\partial^{2} x_{3} / \partial x_{2}^{2}\right)-\left(\partial^{2} x_{3} / \partial x_{1} \partial x_{2}\right)^{2}}{\left(1-\left(\partial x_{3} / \partial x_{1}\right)^{2}-\left(\partial x_{3} / \partial x_{2}\right)^{2}\right)^{2}}
$$

we obtain (16).

Now, from (17), it is not difficult to prove that the graph $(u, v, \phi(u, v))$ gives rise to an immersion into $\boldsymbol{R}^{3}$, which has conformal second fundamental form. 
Conversely, given a solution $\phi$, the harmonic map $N$ can be recover by the inverse Legendre transformation of the graph $(u, v, \phi(u, v))$. That is, the spacelike immersion with constant negative Gaussian curvature -1 given by $\left(\phi_{u}, \phi_{v}, u \phi_{u}+v \phi_{v}-\phi\right)$ agrees with $x$, and $N$ is its Gauss map. Thus, the theorem follows in an easy way.

\section{REFERENCES}

[ 1 ] K. Akutagawa, Harmonic diffeomorphisms of the hyperbolic plane, Trans. Amer. Math. Soc. 342 (1994), 325-342.

[ 2 ] K. AkUtagawa And S. Nishikawa, The Gauss map and spacelike surfaces with prescribed mean curvature in Minkowski 3-space, Tôhoku Math. J. 42 (1990), 67-82.

[ 3 ] T. K. K. AU, L. TAm And T. Y. H. Wan, Hopf differentials and the images of harmonic maps, Comm. Anal. Geom. 10 (2002), 515-573.

[ 4 ] H. Choi And A. Treibergs, Gauss maps of spacelike constant mean curvature hypersurfaces of Minkowski space, J. Differential Geom. 32 (1990), 775-817.

[ 5 ] M. DajCzer and K. Nomizu, On flat surfaces in $\boldsymbol{S}_{1}^{3}$ and $\boldsymbol{H}_{1}^{3}$, Manifolds and Lie groups (Notre Damme, Ind., 1980), 71-108, Birkausser, Boston, Mass., 1981.

[ 6] J. Eells And L. Lemaire, Selected topics in harmonic maps, Reg. Conf. Ser. Math. 50, Published for the Conference Board of the Mathematical Sciences, Washington, DC; by Amer. Math. Soc., Providence RI, 1983.

[ 7 ] J. A. GÁlvez And A. Martínez, The Gauss map and second fundamental form of surfaces in $\boldsymbol{R}^{3}$, Geom. Dedicata 81 (2000), 181-192.

[ 8 ] B. GUAN, The Dirichlet problem for Monge-Ampère equations in non-convex domains and spacelike hypersurfaces of constant Gauss curvature, Trans. Amer. Math. Soc. 350 (1998), 4955-4971.

[9] J. Hano And K. Nomizu, On isometric immersions of the hyperbolic plane into the Lorentz-Minkowski space and the Monge-Ampère equation of a certain type, Math. Ann. 262 (1983), 245-253.

[10] J. Jost, Compact Riemann surfaces, Springer-Verlag, Berlin, 1997.

[11] O. Kobayashi, Maximal surfaces in the 3-dimensional Minkoski space $\boldsymbol{L}^{3}$, Tokyo J. Math. 6 (1983), 297309.

[12] O. Kobayashi, Maximal surfaces with conelike singularities, J. Math. Soc. Japan 36 (1984), 609-617.

[13] A. M. LI, Spacelike hypersurfaces with constant Gauss-Kronecker curvature in the Minkowski space, Arch. Math. 64 (1995), 534-551.

[14] P. Li AND L. F. TAM, The heat equation and harmonic maps of complete manifolds, Invent. Math. 105 (1991), $1-46$.

[15] T. K. MiLnOR, Harmonic maps and classical surface theory in Minkowski 3-space, Trans. Amer. Math. Soc. 280 (1983), 161-185.

[16] E. A. Ruh And J. Vilms, The tension field of the Gauss map, Trans. Amer. Math. Soc. 149 (1970), $569-573$.

[17] R. SCHOEN AND S. YAU, On univalent harmonic maps between surfaces, Invent. Math. 44 (1978), $265-278$.

[18] Y. ShI, L. TAM AND T. Y. H. WAN, Harmonic maps on hyperbolic spaces with singular boundary value, J. Differential Geom. 51 (1999), 551-600.

[19] A. E. TREIBERGS, Entire spacelike hypersurfaces of constant mean curvature in Minkowski space, Invent. Math. 66 (1982), 39-56.

[20] T. Y. H. WAN, Constant mean curvature surface, harmonic maps and universal Teichmüler space, J. Differential Geom. 35 (1992), 643-657.

[21] T. Y. H. WAn And T. K. K. AU, Parabolic constant mean curvature spacelike surfaces, Proc. Amer. Math. Soc. 120 (1994), 559-564. 
[22] T. Weinstein, An introduction to Lorentz surfaces, de Gruyter Exp. Math. 22, Walter de Gruyter, Berlin, New York, 1996.

DEPARTAMENTO DE GeOMETRía y TOPOLOGía

FACULTAD DE CIENCIAS

UNIVERSIDAD DE GRANADA

18071 GRANADA

SPAIN

E-mail addresses: jagalvez@ugr.es

amartine@ugr.es

milan@ugr.es 